\title{
MEDELLÍN, UNA CIUDAD TRANSFORMADA POR SU GESTIÓN. ANÁLISIS DE LA GESTIÓN DE SU CENTRO HISTÓRICO [1990-2015] $]^{(*)}$
}

\author{
MEDELLIN, A CITY TRANSFORMED BY ITS MANAGEMENT. \\ ANALYSIS OF THE MANAGEMENT OF ITS HISTORICAL CENTER [1990- 2015] \\ DÉBORA ARADÍ NÚÑEZ CORONEL ${ }^{[\star]}$ \\ Universidad Nacional Mayor de San Marcos (Perú) \\ dnunezcoronel@gmail.com \\ Fecha de recepción: 01 de abril de 2018 \\ Fecha de aprobación: 08 de diciembre de 2018
}

\section{RESUMEN}

El presente documento busca realizar un análisis sobre la gestión de los centros históricos colombianos, cuya relevancia se observa a partir de su eficacia en sus gestiones actuales. La presente investigación tiene como personaje principal a la ciudad de Medellín, donde se han realizado intervenciones específicamente durante los últimos 25 años, desde 1990 hasta el 2015. El análisis está enmarcado en el contexto general del patrimonio dentro de Colombia, y recurre a algunos documentos oficiales del gobierno, así como a análisis de los enfoques políticos y patrimoniales, los cuales sirven como base para realizar las estrategias asumidas por el Estado. Todos estos documentos, a partir de los cuales se elaboraron las políticas locales de Medellín, se revisan aquí con el fin de poder conocer a profundidad las estrategias en cuestión. Fue necesaria también la revisión del Plan de Ordenamiento Territorial (POT) local, el cual busca la conservación del patrimonio y el desarrollo de las ciudades, articulado al Plan de Desarrollo Municipal (PDM), parte de la estrategia del gobierno para la mejora integral de los barrios.

\section{PALABRAS CLAVE}

Medellín; centro histórico; gestión.

\begin{abstract}
The investigation seeks to carry out an analysis of the management of Colombian historical centers, relevant due to the efficacy of their current management. It has as its main character the city of Medellin, on which interventions have been carried out specifically during the last 25 years, from 1990 to 2015. The analysis is framed within the general context of heritage within Colombia, and includes the examination of some official government documents, and some analysis of the political and patrimonial approaches on which the strategies undertaken by the state are based on. All of these documents, used to create the local policies of Medellín, were reviewed, in order to be able to know the employed strategies in depth. It was also necessary to review the local Plan of Territorial Organization (POT), which pursues heritage conservation and city development, and is articulated in the Municipal Development Plan (PDM), both of which are part of the government's strategy for the comprehensive improvement of neighborhoods.
\end{abstract}

\section{KEYWORDS}

Medellín; historic center; management

(*) Este estudio es parte de un trabajo de investigación desarrollado en el seminario Rehabilitación, Revitalización y Gestión de las Ciudades Históricas (ciclo 2017-II), dictado por el MSc. Arq. José Hayakawa Casas en la Maestría de Gestión del Patrimonio Cultural de la Unidad de Posgrado de Ciencias Sociales de la Universidad Nacional Mayor de San Marcos (UNMSM).

${ }^{(* *)}$ Bachiller en Teología por la UniCesumar en Brasil, estudiante de la Maestría de Gestión del Patrimonio Cultural de la Unidad de Posgrado de Ciencias Sociales de la Universidad Nacional Mayor de San Marcos. Es especialista en temas de educación patrimonial. Profesionalmente, se ha dedicado en diferentes instituciones educativas y ONG que trabajan con la temática del patrimonio. Email: d.nunezcoronel@gmail.com 
devenir Vol. 6, N¹1, ENERO - JUNIO 2019, PP. 69-86 - ESTUDIOS I ISSN 2312-7562 I E-ISSN 2616-4949

UNIVERSIDAD NACIONAL DE INGENIERÍA, LIMA

doi: https://doi.org/10.21754/devenir.v6i11.634

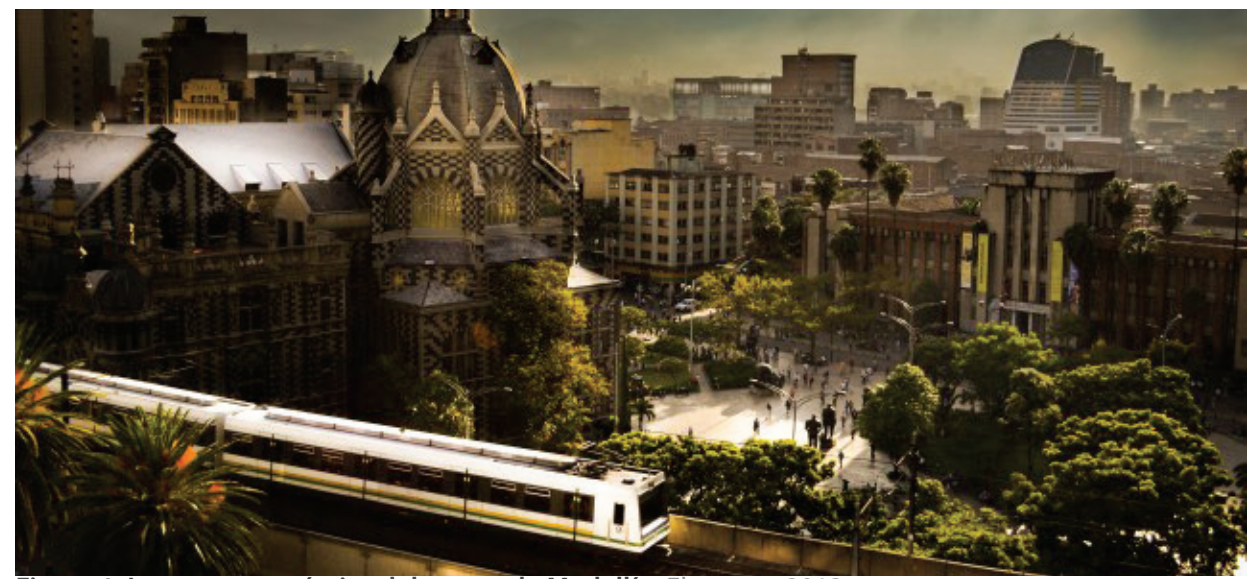

Figura 1. Imagen panorámica del centro de Medellín. El museo, 2013.

\section{Antecedentes de la ciudad}

La ciudad de Medellín es interesante no solo por la historia que alberga, sino por el cambio que experimentó en los primeros 50 años del siglo XX. Pasó de ser un pueblo grande a una ciudad, lo que generó un crecimiento desordenado. Durante las siguientes décadas, hacia los años 80 , aumentaron el crimen organizado, el tráfico de drogas y la delincuencia. No obstante, a principios del s. XXI la ciudad estaba ya completamente transformada.

La metamorfosis que ha atravesado en los últimos 25 años ha convertido a Medellín en una ciudad con una nueva cara, la cual ha sido producto de una buena organización, educación y ganas de los ciudadanos de cambiar.

La ciudad de Medellín asumió la crisis como un aliado para la transformación. Una nueva historia fue contada desde aquellas décadas de 1950 hasta la actualidad. Por ejemplo, en 2012, Medellín fue reconocido como una ciudad que utilizaba transporte sustentable, lo que realzó su título tradicional de capital cultural de Colombia. Así mismo, en 2013, fue reconocida internacionalmente como la ciudad innovadora del año. Del mismo modo, durante los últimos 15 años, se han construido bibliotecas, escuelas y parques en los barrios pobres más alejados. El alcalde Aníbal Gaviria, en una entrevista, reconoció lo siguiente: "Somos innovadores en el desarrollo urbano y también en el desarrollo de programas sociales. Una ciudad que, a pesar de sus problemas en el pasado, se reconstruye constantemente y se ha convertido en una fuente de inspiración para otras naciones" (Camargo, 2013).

Al considerar el cambio que atravesó Medellín, resulta evidente la importancia de una sociedad que desea el cambio, así como de una adecuada planificación urbana de parte de los municipios y la articulación a un plan nacional de desarrollo, para alcanzar el grado de conservación del patrimonio y desarrollo que en muchas ciudades se aspira lograr. En ese sentido, la ciudad de Medellín ha pasado del caos a la esperanza (Figura 1).

\section{Marco metodológico}

El presente trabajo se desarrolló por medio de la recopilación bibliográfica de los documentos empleados por la gestión ciudadana de Medellín entre 1990 y 2015, y se realizó un análisis de planteamientos para la gestión de los últimos 30 años. A partir de esta recopilación, se reconoció que la ciudad de Medellín ha pasado por tres importantes momentos en su planificación: un primer momento, entre los años 1990 y 2003, agobiado por el dolor; un segundo momento, caracterizado por el plan de 2004-2011, 
iluminado por la esperanza; y un tercer momento, entre 2012 y 2015, habitado por la vida. Precisamente este último será objeto de estudio de la presente investigación.

Para este análisis, se planteó primero realizar un marco de estudio general de Colombia, revisando documentos oficiales del Municipio de Medellín. Este análisis abarcó los contextos geográficos, políticos, sociales y económicos de este país, ya que los lineamientos de la nación al respecto son de gran importancia para entender las políticas específicas de cada municipio en el área cultural y, así, poder comprender los trámites y procedimientos involucrados en la ejecución de las actividades en sus centros históricos. Analizar este ámbito permitirá conocer la visión que el Estado posee sobre patrimonio y, específicamente, sobre los centros históricos en Colombia.

También, se revisan algunas investigaciones de expertos nacionales, como Rosa Ramos, cuyo artículo sobre las políticas públicas colombianas para la protección del patrimonio cultural es interesante. Luego de estos análisis sobre las políticas, gestiones y administraciones en Colombia con referencia a la cultura, se finalizará con una discusión sobre las labores realizadas en el Centro Histórico de Medellín y las correspondientes conclusiones.

Este análisis mostrará el camino recorrido por Colombia para llegar a lo que es hoy. Por ello, se analizará cuáles fueron los lineamientos, las leyes y las actividades necesarias para llevar a cabo esta trascendental transformación, ya que es relevante conocer cómo fueron resueltos, para inicios del siglo XXI, aquellos problemas que atormentaron a Medellín durante la segunda mitad del siglo pasado.

\section{Marco teórico}

La gestión de Medellín, desde los años 90 hasta 2015, basó su visión en relación a la gestión de centros históricos en la definición planteada en el Coloquio de Quito de 1977, cuyo tema fue La Preservación de los Centros Históricos ante el Crecimiento de las Ciudades Contemporáneas. Este coloquio representó un gran acontecimiento para la gestión de centros históricos de América Latina. En esta reunión, se definió como centro histórico

Todos aquellos asentamientos humanos vivos, fuertemente condicionados por una estructura física proveniente del pasado, reconocibles como representativos de la evolución de un pueblo. Como tales se comprenden, tanto asentamientos que se mantienen íntegros, desde aldeas o ciudades, como aquellos que a causa de su crecimiento, constituyen hoy parte o partes de una estructura mayor. (UNESCO, 1977)

Esta definición permite observar un aspecto importante: la dimensión de ciudad se ubica antes que la dimensión de patrimonio. Esa es la combinación perfecta para poder gestionar un centro histórico, ya que este debe ser primero contemplado como ciudad, como una ciudad que vela por los intereses de sus habitantes sin dejar atrás su valor histórico.

Al ser un asentamiento vivo, el centro histórico requiere de una mirada más integral para ser gestionado. Esto se debe a que no solo debe ser vista desde una mirada patrimonial, para la que lo único importante son los monumentos, sino que el enfoque debe dar importancia a la urbanidad, que implica ver al ser humano como aquel que puede disfrutar de estos lugares con valor patrimonial, lo cual mejora su calidad de vida.

La gestión de un centro histórico debe contemplar intervenciones que consideren al centro histórico en tanto ciudad, y no simplemente como un espacio que contiene bienes de valor patrimonial.

El centro histórico es un lugar de encuentro entre personas. Diariamente, personas de diferentes condiciones recorren ese espacio, ya sea por trabajo o por visita. Es precisamente allí donde estas diferencias se diluyen y las personas se relacionan. Por ello, es 




Figura 2. Ubicación de Colombia en América del Sur. Tindo, 2018.

posible afirmar que el centro histórico es un espacio vivo. Una vez que se pierde esta dimensión, solo queda un centro histórico con bonitas fachadas antiguas, que solo sirven para sacar buenas postales. En el documento Plan de Gestión Integral de la Intervención del Centro: Centro Metropolitano de Medellín, una Construcción desde lo Territorial, la Planeación y el Imaginario (PGIIC), empleado para la intervención en este centro, se declara literalmente que el objetivo de esta intervención es el siguiente:

Mejorar las condiciones de calidad de vida de los habitantes y transeúntes del Centro de la ciudad de Medellín, la calidad urbanística y ambiental del Centro, y la recuperación de su significado y convocatoria para todos los sectores sociales de la ciudad y el área metropolitana, mediante la integración coherente de las diversas intervenciones en gestación o en marcha a su interior o en su periferia inmediata, y la protección y potencialización de su patrimonio cultural. $(2015$, p. 7$)$

Dicho esto, la intervención integral es fundamental para la solución de los conflictos presentes en este espacio de la ciudad, que permita buscar la participación activa de la sociedad.

\section{Un análisis de Colombia}

Colombia es un país geográficamente muy diverso, diversidad que a su vez ha generado gran variedad natural y cultural. Estas características lo señalan como un territorio de gran riqueza patrimonial, al igual que muchos países de América Latina. 


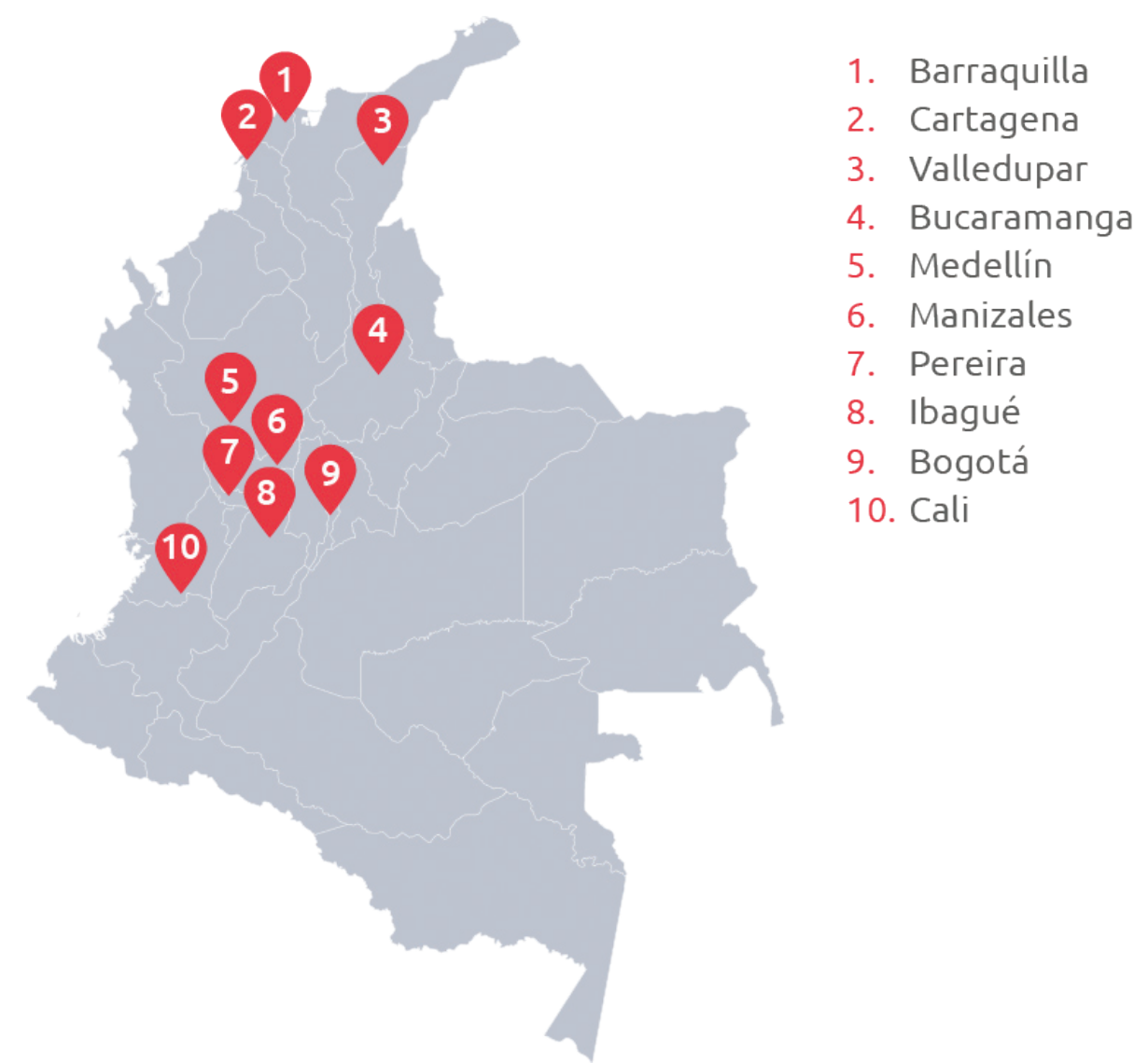

Figura 3. Principales ciudades de Colombia que en los últimos años han experimentado un gran cre-cimiento. Humanaun Colombia, 2018.

Colombia está situada en la esquina noroeste de América del Sur. Su territorio se extiende a lo largo de ambos hemisferios, norte y sur. Su territorio continental abarca $1^{\prime} 141.748 \mathrm{~km}^{2}$ y su extensión marítima asciende a 2'129.748 km². Esto lo convierte en un país de inmensurable riqueza y biodiversidad. La biodiversidad natural que alberga la ubica el segundo lugar entre los 12 países más megadiversos del planeta (Colciencias, 2018) (ver Figura 2).

Así mismo, la biodiversidad étnica de este país es interesante. Según el Departamento Administrativo Nacional de Estadísticas (DANE), en 2005, las personas que se consideraban blancos componían el $85.9 \%$ de la población; afrocolombianos, $10.62 \%$; indígenas, $3.43 \%$; y rumanos, $0.01 \%$. Esto convierte a Colombia en un país muy diverso.

El territorio colombiano está dividido en seis regiones naturales: andina, pacífica, amazónica, Orinoquia, insular y Caribe. Debido a la riqueza mineral de los pisos térmicos del norte del país, es ese el lugar que se volvió llamativo para vivir, ya que allí se desarrollan las principales actividades económicas, como la ganadería, la agricultura, el turismo y la minería. Por esa razón, todo el poder económico se concentra en las principales ciudades. Esa es la razón por la cual una parte de Colombia está más densamente habitada: solo en la zona norte del país, se encuentra el $75 \%$ de la población colombiana (ver Figura 3).

Colombia cuenta con cinco importantes ciudades: Medellín, Bogotá, Cali, Barranquilla y Cartagena. Es importante resaltar este aspecto, ya que es entre estas ciudades 
devenir Vol. 6, N¹1, ENERO - JUNIO 2019, PP. 69-86 - ESTUDIOS I ISSN 2312-7562 I E-ISSN 2616-4949

UNIVERSIDAD NACIONAL DE INGENIERÍA, LIMA

doi: https://doi.org/10.21754/devenir.v6i11.634

que se divide la mayor parte de la población; no solo una está sobrecargada con una superpoblación.

En cuanto a temas políticos, Colombia es un Estado unitario, social y democrático, cuya forma de gobierno es presidencialista. Es una república que está organizada en 32 departamentos descentralizados, cuya capital es Bogotá, sede del gobierno nacional. La Constitución Política de Colombia ordena el poder público en tres poderes del Estado: ejecutiva, legislativa y judicial. De esta manera, se busca evitar la concentración del poder. Cada rama cumple diferentes funciones y actúa de forma independiente pero armónica, según la constitución.

Cabe puntualizar que el tema patrimonial en Colombia adquirió un papel preponderante en el panorama cultural del país. Evidentemente, se ha recorrido un gran camino desde sus comienzos, en el año 1959, cuando se promovió la Ley Nㅜ둘, por medio de la cual se dictaron medidas de defensa y conservación del patrimonio nacional. Es en 1997 cuando ocurre un hecho significativo para Colombia y su cultura: la Ley №397, la Ley General de Cultura, es aprobada. Esta fue nutrida con leyes y decretos fundamentales para la preservación y gestión del patrimonio colombiano.

En Colombia, el ente que está encargado de las políticas en el sector cultural es el Ministerio de Cultura. Al igual que en el Perú, el trabajo principal desempeñado por esta entidad es promover, conservar y divulgar el patrimonio cultural, junto con las entidades adscritas, los organismos vinculados a este, y organismos autónomos vinculados a otros ministerios y entidades afines, los cuales son los encargados de formular, ejecutar y supervisar las políticas culturales.

En otras palabras, es el Ministerio de Cultura el que adquiere la responsabilidad de administrar el ámbito cultural en Colombia, labor para la cual cuenta con el apoyo de otras organizaciones. Gracias a ello, el trabajo que ha sido realizado ha resultado exitoso, ya que se llevó a cabo de forma multidisciplinaria. Todo lo contrario se aprecia en el caso peruano: el Ministerio de Cultura no logra poder cuidar y gestionar todos los bienes, y, más aun, no está articulado con otros ministerios o entidades para la protección de los bienes culturales.

\section{Historia de la fundación de Medellín}

Medellín surge en medio de un conflicto de intereses entre dos ciudades: Santa Fe de Antioquía y Rionegro, las cuales estaban en contra de la creación de Medellín, por restarles protagonismo. Por esta razón, la creación de esta ciudad sucedió 10 años después de su supuesto planeamiento. Debido a una importante suma de intereses, en 1675, se creó bajo el nombre Villa de Nuestra Señora de la Candelaria. Muchos años después, recién en 1913, recibiría el título de ciudad.

Esta ciudad fue fundada cerca de ríos, en tierras muy fértiles, donde abundaron la ganadería, la minería y los cultivos de caña, una zona donde burgueses adinerados poseían muchas propiedades. Su ritmo acelerado de crecimiento económico llamó la atención de mucha gente, que decidió migrar a esta nueva villa. Años después de su creación, en el censo de 1786, realizado en el gobierno de Mon y Velarde, se enumeraron las calles de Medellín, se separaron los barrios de la ciudad y se registraron hasta 45 manzanas y 241 casas.

En 1826 se asignó como capital de Medellín a Antioquía, hecho que convirtió a esta ciudad en una mucho más atractiva, por su crecimiento económico. Todo ello permitió que en 1850 su población ascendiera al doble (ver Figura 4).

A inicios del siglo XX, las actividades económicas tomaron un nuevo camino. Por ejemplo, todas las actividades agrícolas se vieron opacadas por las actividades indus- 


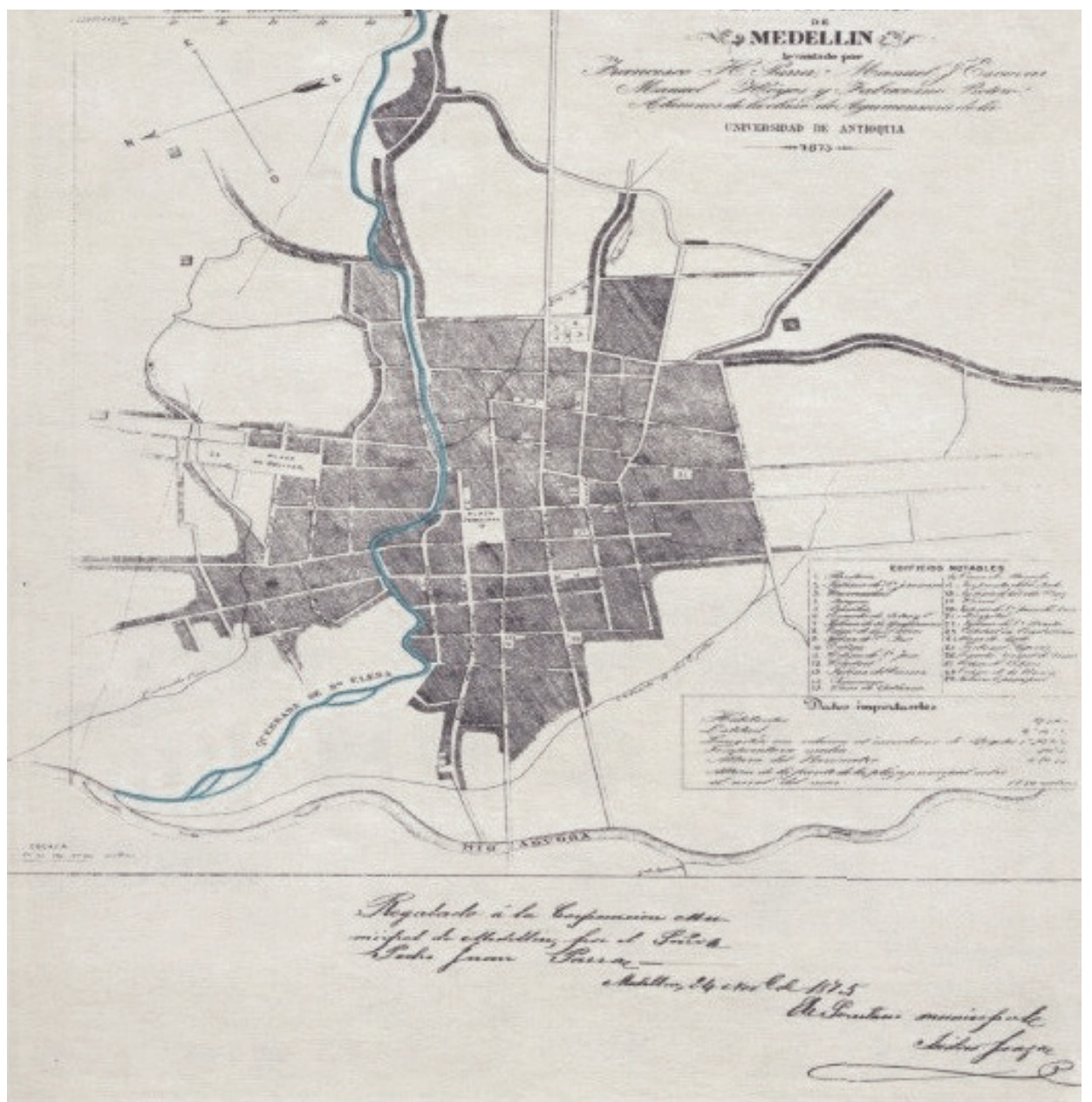

Figura 4. El presente plano corresponde a la ciudad de Medellín en 1875. Biblioteca Pública Piloto, 2018.

triales, lo cual atrajo a más personas interesadas en el crecimiento económico de la ciudad. Este crecimiento exagerado condujo a que se considere necesario planificar su territorio y a tratar de organizarlo. Entre los grandes proyectos de modernización y organización que se llevaron a cabo, se puede mencionar algunos principales, como la canalización de la quebrada Santa Helena, construida en 1930; y la ratificación y canalización del río Medellín en 1943. Durante estas décadas, la ciudad experimentó un crecimiento súper acelerado. Se registró un crecimiento de $5.8 \%$ anual. Medellín estaba creciendo desproporcionalmente, y hacia los años 40 todo estaba en absoluto caos. Todos los servicios estaban fuera de la zona urbana y las edificaciones estaban en muy mal estado. Por esta razón, Medellín necesitaba urgentemente un plan piloto para la ciudad, que fue elaborado en 1948 por los arquitectos Paul Wiener y José Luis Sernt (Melo, 1996).

El plan realizado en 1950 es uno de los planes más importantes de la ciudad, ya que fue un plan regulador que "ofrecería directrices generales para la reorganización de la ciudad y su crecimiento, y tenía en cuenta sus particularidades y su ubicación en un valle de gran belleza" (Perfetti, 1996, p. 215).

Desde inicios del siglo XX hasta la mitad del mismo, Medellín pasó de ser un pueblo grande a una ciudad importantísima, donde se desarrollaban las principales actividades económicas, sociales, políticas y culturales de la región de Antioquía. 
devenir Vol. 6, N¹1, ENERO - JUNIO 2019, PP. 69-86 - ESTUDIOS I ISSN 2312-7562 I E-ISSN 2616-4949

UNIVERSIDAD NACIONAL DE INGENIERÍA, LIMA

doi: https://doi.org/10.21754/devenir.v6i11.634

Para 1973 se creó la Oficina de Planeación Metropolitana. Esta iniciativa reflejó que, para hacer frente al crecimiento urbano, se asumió una responsabilidad estratégica de parte de la propia ciudad. Desde ese entonces, el desarrollo urbanístico tomó un rumbo distinto y se comenzó a actuar desde una mirada interdisciplinaria.

En 1985 se armó un nuevo plan para la consolidación de la metrópoli. Sin embargo, no se obtuvieron los resultados esperados. En los años 90, Medellín recibió una ola de migrantes que llegaban del campo a la ciudad en busca de un mejor futuro. Estas personas se asentaron en las faldas de los cerros cercanos a la ciudad, lo que ocasionó que esta resultase dividida: en el Sur, existían todos los equipamientos que una ciudad necesita, como parques, avenillas, calles; en el Norte, la gente vivía aglomerada sin la mínima noción de una vida urbana ideal. Precisamente en esa misma década se vuelve a crear un plan, a partir de legislaciones nacionales, orientado a unir estas dos realidades de Medellín.

En 1993 Pablo Escobar, el más grande narcotraficante de Colombia, fue asesinado. Con ello, la red de narcotráfico arraigada en Medellín comenzó a perder territorio, y entonces comenzó a abrirse paso un cambio por el cual se había estado trabajando. Por esos mismos años, se promulga la ley más importante para la legislación colombiana respecto del ámbito de la cultura, la Ley №397 de 1997, por la cual se crea el Ministerio de Cultura.

Estas décadas fueron de suma importancia para el desarrollo de Medellín: lo que tuvo lugar durante ese periodo permitió que, a inicios del año 2000, se comience a elaborar los planeamientos para el ordenamiento territorial, necesario para organizar mucho mejor la ciudad.

\section{Políticas públicas sobre patrimonio en Colombia}

Desde inicios del siglo XX, el gobierno colombiano ha estado interesado en cuidar su patrimonio. Esto se refleja en el hecho de que, con el pasar del tiempo, se ha producido variada legislación sobre el patrimonio nacional. Ello es muestra de la preocupación y responsabilidad que el gobierno asume con respecto al patrimonio, porque el desarrollo legislativo está caracterizado por la adhesión a tratados y convenios internacionales desde la década de los años 30 (Garavito, 2006).

El interés del Estado por el patrimonio comienza a inicios del siglo XX, y toma forma más sólida hacia 1959, con la expedición de la Ley № 163 . En esta se puede apreciar los inicios de los cuidados políticos del patrimonio. Esta ley fue la antecesora de la promulgación de la Constitución Política de 1991 y de la Ley General de Cultura. Así el Estado generó un nuevo marco general de actuación, al reconocer el carácter multiétnico y pluricultural de la Nación.

Una de las competencias del Estado colombiano en temas culturales consiste en que el Ministerio de Cultura, por medio de los museos, asume la responsabilidad del cuidado del patrimonio y toma como su deber, por un lado, incentivar a que más museos de diferentes áreas permitan el libre acceso y, por otro lado, promover que se realicen investigaciones y publicaciones por medio de la Biblioteca Nacional.

Además del Ministerio de Cultura, existen algunas instituciones adscritas a este, como ya se mencionó anteriormente. Entre las principales está el Instituto Colombiano de Antropología e Historia (ICANH), el cual se encarga de difundir y preservar el patrimonio colombiano; también el Archivo General de la Nación, al cual le corresponde elaborar y mantener actualizado el registro del patrimonio monumental (Ministerio de Cultura, 2013).

Como se aprecia, el enfoque desde el cual se trabaja es multidisciplinario, pues todos los ministerios están íntimamente relacionados. En Colombia, el tema cultural está 
muy articulado al urbanismo; uno de los antecedentes más importantes es la Ley de la Reforma Urbana, aprobada en 1989. Con ella nació el derecho urbanístico de los colombianos, aunque recién en 1991 se logró pulirla, y entonces se generaron los primeros conceptos que reconocen el derecho al uso del espacio público. Estas dos leyes, la de derecho urbanístico y la del uso del espacio públicos, son de vital importancia para el patrimonio y la intervención en este en las ciudades históricas.

Se consolidó así, como parte de los derechos colectivos, el derecho al uso y goce del espacio público. Se convirtió en una preocupación de orden constitucional, que ha sido objeto de un amplio desarrollo jurisprudencial. De igual modo, la Constitución introdujo en 1991 el derecho a una vivienda digna y al acceso a los servicios públicos. Este derecho luego se vería plasmado en la gestión del Centro Histórico de Medellín, donde esta política ayudó a que se transforme en un lugar donde se pueda vivir con dignidad, y no solo sea visto como un conjunto de fachadas antiguas bien cuidadas. Cabe resaltar, en este punto, la importancia de la preocupación del Estado por el bienestar de las personas, más que por los monumentos. Esto se debe a que son las personas quienes hacen que un centro histórico esté vivo; si no hubiesen personas viviendo en este espacio, el centro sería como el escenario de una película de la Edad Media, donde es imposible no tomarse una foto, pero cuyo momento desaparece al ocultarse el Sol. Esta actitud en un centro histórico permite que la denominación de ciudad se extinga, pues solo es ciudad aquel lugar donde existe una sociedad.

Una de las leyes más importantes que se aprobó en Colombia, en 1997, fue la Ley General de Cultura. Esta fue una ley claramente intervencionista, cuyos objetivos y los de sus instrumentos no hubieran podido ser llevados a la práctica sin una decidida actuación del Estado a través de los municipios y distritos, de las autoridades metropolitanas de planeación, y de las autoridades ambientales dirigidas a redefinir el derecho de propiedad y a configurar una nueva manera de hacer planeación y gestión urbanas (Pinilla, 2003).

La Ley General de Cultura tiene una agenda muy cargada respecto de la legislación, pues obliga al Estado a la protección, conservación, rehabilitación y divulgación del patrimonio cultural, y a coordinar labores que antes habían desempeñado independientemente distintas instituciones públicas y privadas. La novedad está en el nuevo marco institucional constituido por el Ministerio de Cultura, y en la obligatoriedad de incorporar el asunto al Plan Nacional de Desarrollo y a los planes de desarrollos departamentales y municipales. A su vez, mediante la Ley $N^{\circ} 388$ o Ley de Planificación Urbana, se estableció la obligatoriedad de elaborar un Plan de Ordenamiento Territorial (POT) en todos los municipios del país. Así mismo, se buscó articular, por primera vez, la conservación del patrimonio y el desarrollo de las ciudades, así como contextualizar el patrimonio inmueble dentro de la totalidad del patrimonio cultural y fortalecer sus vínculos con los modos de vida de las comunidades.

La intervención por parte del gobierno se efectúa a través de los municipios que mantengan o estén ejecutando proyectos de mejora integral de barrios, llamados también Plan de Desarrollo Municipal (PDM), y renovación urbana. Precisamente, a estos municipios el gobierno brinda soporte técnico, acompañamiento, capacitación y todas las herramientas necesarias para que estos proyectos se desarrollen exitosamente, con el fin de adelantar la prevención de desastres, riesgo, vivienda, infraestructura, entre otros (Ministerio de Vivienda, Ciudad y Territorio, 2014). Todos estos han sido reglamentados por la Ley N ${ }^{\circ} 152$ de 1994, que permitió un elaborar un Plan de Desarrollo (Rodríguez, 2015).

Por lo tanto, se podría afirmar que los municipios colombianos son independientes al planificar, pero están empoderados por parte del gobierno al momento de actuar. Cuando aparecieron los POT, hace más de 18 años atrás, cada ciudad elaboró una lista de sus bienes con valor patrimonial, ligada a su plan de desarrollo integral municipal, 
devenir Vol. 6, N¹1, ENERO - JUNIO 2019, PP. 69-86 - ESTUDIOS I ISSN 2312-7562 | E-ISSN 2616-4949

UNIVERSIDAD NACIONAL DE INGENIERÍA, LIMA

doi: https://doi.org/10.21754/devenir.v6i11.634

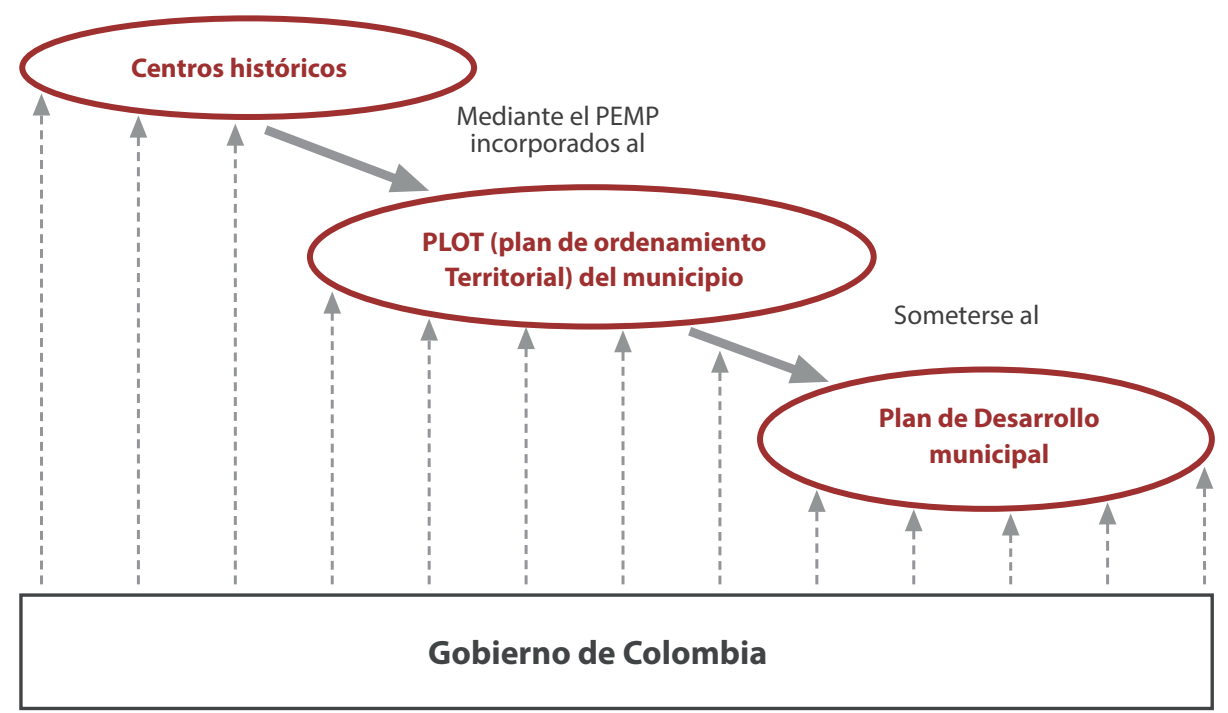

Figura 5. Mecanismos que usa el gobierno para gestionar los centros históricos. Elaboración propia sobre la base del artículo de Diana Ramos en la revista Devenir 6, 2018.

y creó cada una un Plan Especial de Manejo y Protección (PEMP), destinado exclusivamente a salvaguardar el patrimonio.

En un artículo interesante de Diana Ramos (2017), “El Plan de Ordenamiento Territorial y el Plan Especial de Manejo y Protección del Patrimonio en los Centros Históricos de Colombia: El Caso Popayán", publicado en la revista Devenir, dicha autora señala que han tenido algunas divergencias con algunas políticas que deberían trabajar en armonía, como es el caso de los POT y los PEMP, ya que un centro histórico mediante su PEMP debería insertarse en el POT del Municipio.

El problema surge cuando los PEMP vulneran la autonomía territorial y la descentralización del POT municipal. Estos dos deben llegar a un acuerdo específico para el bienestar de la ciudad. En otras palabras, el POT, por un lado, vela por un desarrollo integral de la ciudad, y el PEMP, por otro lado, está interesado en el bien patrimonial. Es más, hay muchos temas que no le competen al PEMP, ya que no incorpora una mirada panorámica de ciudad, sino más bien una mirada específica sobre un determinado bien, pues el PEMP, por ser una norma de mayor jerarquía, obliga a los POT a incluirlo dentro de sus planes. Los POT, por su parte, tampoco cuentan con un plan efectivo para el cuidado de los bienes de interés cultural. Entonces, resulta evidente que el problema llega cuando estos dos no se corresponden (Ramos, 2017)(ver Figura 5).

El Consejo de Monumentos Nacionales de Colombia, en su calidad de órgano asesor, está encargado de orientar al Gobierno Nacional y al Ministerio de Cultura en el diseño de las políticas estatales en cuanto al patrimonio cultural, con el objetivo de protegerlo, conservarlo, rehabilitarlo, y divulgar su valor. Este trabajo es fundamental para que una gestión cultural pueda ser llevada a cabo. Colombia es uno de los países con mayor diversidad cultural, y uno de los cuales trabaja bastante en esta área. Por ende, este órgano asesor es de suma importancia para el desarrollo cultural de este país.

Para mejorar el manejo y protección en las intervenciones en los centros históricos, se expidió la Ley № 11885 en 2008, y se elaboraron tres decretos relativos al Consejo Nacional de Patrimonio Cultural, al Patrimonio Cultural de la Nación Material y al Patrimonio Cultural de la Nación Inmaterial. Cada uno de los lineamientos está compuesto por estrategias para actuar involucrando a los municipios locales, distritales y departamentales, todos ellos enmarcados dentro de los planes de la dirección de patrimonio (Ramos, 2017). 
Otro órgano fundamental en el ámbito cultural es el Consejo Nacional de Áreas Protegidas (CONAP), un comité asesor del ICANH, una institución de carácter científico y técnico, sin fines de lucro, creado mediante Resolución 0282 del 3 de abril de 1997 por COLCULTURA, con el propósito de fijar parámetros de implementación, ejecución y evaluación de estudios sobre impacto de obras de infraestructura al patrimonio arqueológico para elevar, y controlar la calidad científica y técnica de dichos estudios, e igualmente para preservar el Patrimonio Cultural de la Nación. Todas estas entidades en los últimos años han contribuido a generar una mejora significativa en Colombia, cuya labor en materia de intervención en el patrimonio se ha desarrollado de forma asombrosa. Como se aprecia, los asuntos vinculados a la cultura han sido en gran medida impactados y beneficiados por las leyes de ese país.

\section{La Gestión del Centro Histórico}

El plan de intervención que aquí se analizará es parte de un proyecto de intervención por medio del POT, realizado entre 2012 y 2015, que consistió en una intervención integral en el centro de la ciudad. Comenzó como un programa bandera del plan de desarrollo, con el propósito de mejorar las condiciones socioambientales, culturales, urbanísticas, de seguridad y de movilidad del Centro Metropolitano, así como de reasignar la capacidad de convocatoria del centro tradicional y representativo, para fortalecer la convivencia, y lograr un centro próspero y apto para caminar y disfrutar, donde el ciudadano se apropie del territorio. Es así como este plan de gestión inició un proceso articulador e integrador de la oferta social, física, cultural, económica y ambiental de los diferentes entes del nivel central y descentralizado para el centro.

Según el Centro Internacional de Estudios para la Conservación y la Restauración de los Bienes Culturales (ICCROM, 2006), en Colombia los centros históricos son los sectores urbanos antiguos que se han desarrollado de forma más o menos homogénea desde la fundación de una ciudad hasta el siglo XVIII o principios del siglo XIX. Este concepto está basado en las Normas de Quito de 1967. En algunos casos, el área urbana es toda el área de los centros históricos, como es el caso de Santa Fe de Antioquia. En otras ciudades, el centro histórico está dentro de la ciudad moderna, como Medellín, Bogotá, Cartagena, Tunja, entre otras.

El Ministerio de Cultura colombiano resalta que Medellín no posee un centro histórico declarado Patrimonio Cultural de la Nación. Eso no significa que el centro de esta ciudad no tenga ningún valor; todo lo contrario, pues en esta se encuentran 30 monumentos de interés nacional, según la lista dada por el gobierno. Según el Ministerio de Cultura (2016), el centro histórico no solo no está declarado, sino que tampoco está delimitado. Para realizar esta intervención, se trabajó en los 23 barrios más antiguos y centrales de la ciudad que contienen espacios de valor patrimonial: Prado, El Chagualo, Jesús Nazareno, Estación Villa, San Benito, La Candelaria, Villa Nueva, Los Ángeles, Boston, Bomboná №1, Corazón de Jesús, Guayaquil, Calle Nueva, Las Palmas, Barrio Colón, El Perpetuo Socorro, San Diego de la Comuna 10, Conquistadores, Naranjal, Carlos E, Restrepo de la Comuna 11, Cerro Nutibara de la Comuna 16, Sevilla y Moravia de la Comuna 4 (ver Figura 6).

El hecho de que su centro histórico no tenga declaratoria de patrimonio ni delimitación fue un punto a favor de la gestión para rehabilitarlo, ya que pudo ser gestionado como parte de la ciudad. Medellín no ha dividido a su ciudad entre centro histórico y ciudad; por el contrario, el primero es considerado parte fundamental de la ciudad y, por lo tanto, se gestiona como parte de un todo, con el principal objetivo mejorar las condiciones de vida de los pobladores. Para eso, se sumaron todas las fuerzas y las áreas a ser trabajadas, para poder tener un resultado integral.

El hecho de que se haya escrito un plan para Medellín no significa que esta ciudad no haya atravesado crisis con respecto a su centro histórico, crisis asociadas al mal uso 


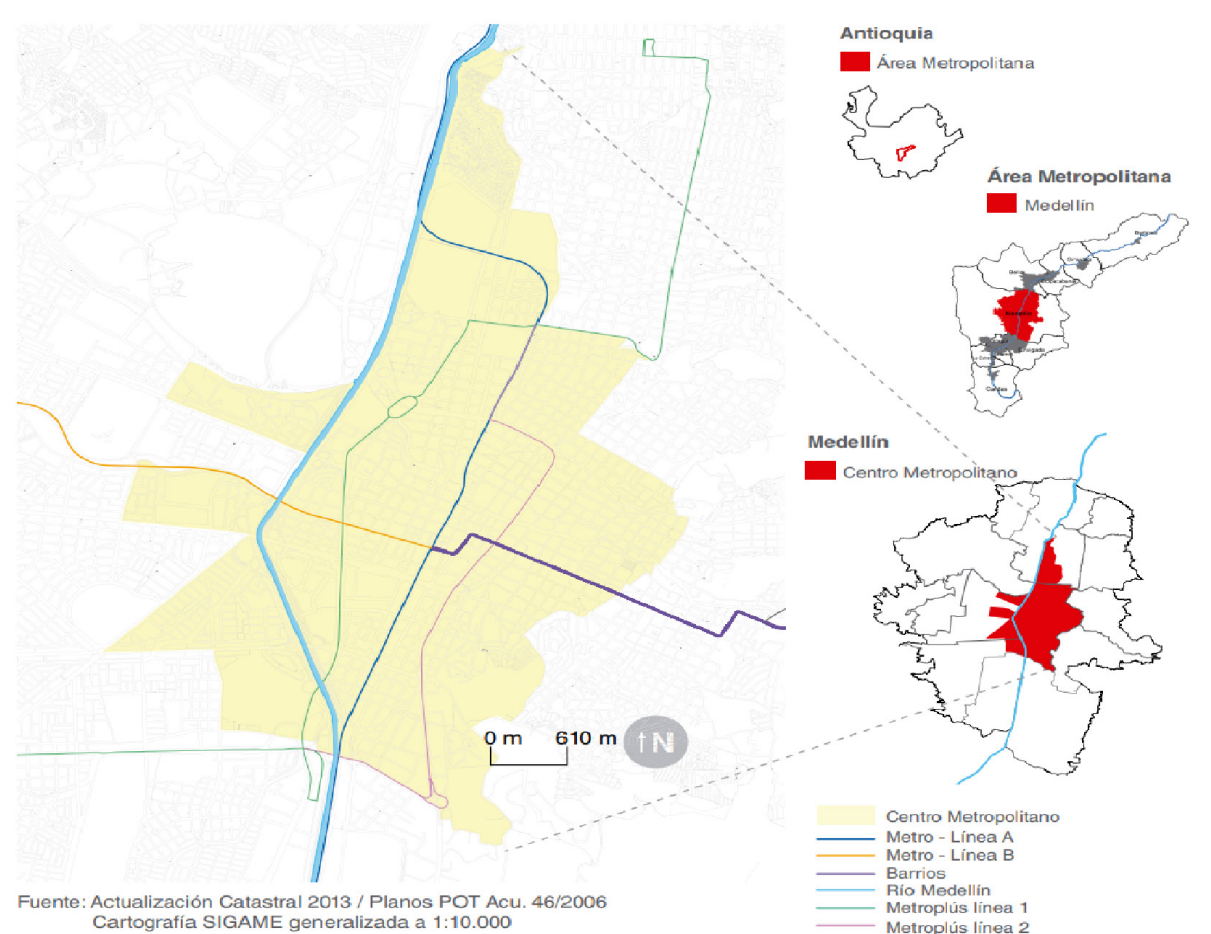

Figura 6. Plano de intervención usada en el plan para gestionar Medellín. Plan de gestión integral de la intervención del centro, 2015.

de las políticas públicas. Con la Ley Urbana de 1989, se empezó a formular planes de desarrollo municipal con un enfoque físico-espacial. Ya comenzando los años 90 , para el uso y ocupación del suelo del centro histórico. En el caso de Medellín, hasta ese entonces el suelo del centro histórico estaba congelado y no estaba incluido en ningún plan. Entonces, comenzaron las presiones tanto de parte de los propietarios como de los inversionistas para realizar en el centro histórico actividades económicas importantes. Muchos de los bienes culturales existentes en el centro resultaban un obstáculo para este proceso, por lo que empezó una destrucción acelerada de estos. Además, las herramientas técnicas de control no eran suficientes, y los llamados opositores procedieron a trasformar los inmuebles patrimoniales con obras de adición y subdivisión, incluso de demolición, para garantizar su aprovechamiento (Rodríguez, 2015). Esto es lo que sucedió en Medellín. Fue más fácil para la presión destruir algunos bienes que no contaban con el título de centro histórico.

Por ello, el historiador Luis Fernando González Escobar (2007) expresa sus ideas acerca de la arquitectura de la ciudad en su libro Memoria y Patrimonio de Medellín.

En Medellín, han reñido tradición y modernidad, el pasado y el futuro. El presente no ha sido un equilibrador entre ambos. Siempre se demuele para progresar con una memoria arquitectónica que no alcanza más de dos generaciones, cuando mucho. Algo de más de treinta años es señalado como antiguo. Basta ver una monografía de 1959 en la que las fotos muestran el supuesto Medellín antiguo y hace referencia a las áreas urbanas con edificios Art Deco de los años 30, esto es, menos de treinta años de haberse construido y eso se consideraba una antigüedad. (p. 36)

Esta intervención estuvo enmarcada en los planes de desarrollo nacional, cuyos principales ejes pueden observarse en el Plan de Gestión Integral de la Intervención del Centro (2012) (Figuras 7 y 8). 




Figura 7. Gráfico del plan de desarrollo nacional 2010-014. Elaborado por Plan de gestión integral de la intervención del centro, 2015.

Estos dos esquemas muestran la estrecha relación entre el plan nacional y el plan municipal para la intervención en un centro histórico. Ambas instituciones mantienen un interés especial por estas actividades. El plan municipal indica actividades de intervención más claras y definidas; entre los tres principales, plantea la necesidad de mejorar, recuperar y potenciar. Estas actividades abarcan otras que tienen una relación íntimamente ligada al POT de cada municipio, que vela no solamente por los edificios con valor patrimonial, sino por la importancia de realizar una intervención integral que contribuya al bienestar social, para recuperar así el significado de los espacios públicos, la calidad urbanística y la calidad ambiental. Estas actividades fueron realizadas por parte de los encargados de la intervención para la recuperación integral del centro de Medellín.

Este plan de desarrollo tuvo como principal objetivo

Mejorar las condiciones de calidad de vida de los habitantes y transeúntes del Centro de la ciudad de Medellín, la calidad urbanística y ambiental del Centro, y la recuperación de su significado y convocatoria para todos los sectores sociales de la ciudad y el área metropolitana, mediante la integración coherente de las diversas intervenciones en gestación o en marcha a su interior o en su periferia inmediata, y la protección y potencialización de su patrimonio cultural. (Alcaldía de Medellín, 2012-2015)

Para la planificación de la recuperación del centro de Medellín, se trabajó con algunas estrategias para una mejora integral del lugar que están íntimamente articuladas al Plan Nacional de Recuperación de Centros Históricos (PNRCH). Así se describió en el Plan de Gestión Integral de la Intervención del Centro:

- Estrategia 1: Rehabilitar el centro

Implica la posibilidad de volver a habitar el centro de nuevo, y que allí se cuente con todo lo necesario para poder hacerlo de manera digna. 
devenir Vol. 6, Nº11, ENERO - JUNIO 2019, PP. 69-86 - ESTUDIOS I ISSN 2312-7562 | E-ISSN 2616-4949

UnIVERSIDAD NACIONAL DE INGENIERÍA, LIMA

doi: https://doi.org/10.21754/devenir.v6i11.634



Figura 8. Gráfico del Plan de desarrollo nacional 2012-2015, Plan de desarrollo municipal. Elaborado por Plan de gestión integral de la intervención del centro, 2015.

- Estrategia 2: Mejorar el espacio público

Apunta a mejorar la experiencia de recorrer y habitar sus sitios urbanos

- Estrategia 3: Fortalecer la presencia institucional

Requiere construir y adecuar infraestructuras que sirvan de apoyo a las acciones de promoción de desarrollo local, económico y social

- Estrategia 4: Incentivar iniciativas públicas y privadas

Estimular proyectos para la gestión e inversión tanto pública como privada, además de promover programas que incorporen el fortalecimiento y sostenibilidad del desarrollo local

- Estrategia 5: Mejorar las condiciones ambientales

Generar acciones orientadas a la recuperación ambiental del centro de la ciudad

- Estrategia 6: Fortalecer la seguridad integral ciudadana

Abordar de manera integral las diferentes problemáticas que se presentan en el ámbito de seguridad y mejorar la convivencia de la ciudadanía

- Estrategia 7: Promover la cultura ciudadana

Fomentar la cultura, el sentido de pertenencia, y el reconocimiento de los derechos, deberes y responsabilidades ciudadanos

- Estrategia 8: Revitalizar el patrimonio urbanístico y arquitectónico

Realizar proyectos de sensibilización respecto de la conservación preventiva, la restauración y la recuperación integral del patrimonio.

Cuando se gestionó la ciudad histórica de Medellín, el Ministerio de Cultura estuvo totalmente involucrado: fue el principal responsable de velar por el patrimonio colombiano, específicamente desde la Dirección de Patrimonio, que en los últimos años ha 




Figura 9. Gráfico del Plan de desarrollo nacional 2010-2014, distribución porcentual de los gastos en esta intervención. Elaborado por Plan de gestión integral de la intervención del centro, 2015.

sido el organismo que revisa y evalúa las políticas del gobierno nacional con el fin de innovar en el cuidado y gestión del patrimonio.

La dirección de patrimonio trabajó con cuatro directrices para lograr el objetivo de que el patrimonio cultural sea asimilado por la ciudad:

1) Grupo de Investigación: Encargado de desarrollar proyectos que aumenten el conocimiento sobre el patrimonio nacional, tuvo como principal tarea realizar la valoración e inventario de los bienes de interés cultural, con el propósito de estructurar las bases documentales e históricas para su posterior protección, intervención y divulgación.

2) Grupo de Intervención: Su tarea consistió en asesorar en la formulación, coordinación, y ejecución de las políticas y los planes generales en materia de intervención en los monumentos nacionales. Cuenta con un equipo especializado en el área de restauración y conservación, que realiza las funciones de supervisión ejecutiva de contratos de obras e intervención.

3) Grupo de Protección: Se encargó de garantizar la conservación de los bienes de interés cultural muebles e inmuebles a través del establecimiento de proyectos y acciones orientados a consolidar un manejo eficiente y sostenible, que incorpore el patrimonio cultural como factor que da estructura al país.

4) Grupo de Formación: Su labor consistió en facilitar el acceso de la población colombiana al conocimiento del patrimonio cultural.

El Grupo de Formación es uno de los más importantes que el Ministerio de Cultura ha generado, ya que este eje es el responsable de llegar a la comunidad e involucrarla con el tema. Una sociedad que no conoce qué están haciendo con su patrimonio no ama ni respeta lo suyo. Partiendo de esta premisa, el papel de este grupo es de vital importancia en el desarrollo de proyectos de cuidado y valoración. Sin el trabajo de este equipo, todas las recuperaciones antes hechas no hubieran tenido el impacto que se ha logrado. 
devenir Vol. 6, N¹1, ENERO - JUNIO 2019, PP. 69-86 - ESTUDIOS I ISSN 2312-7562 | E-ISSN 2616-4949

UNIVERSIDAD NACIONAL DE INGENIERÍA, LIMA

doi: https://doi.org/10.21754/devenir.v6i11.634

Otra institución que estuvo involucrada en este centro histórico es el ICANH, que realiza actividades de investigación en materia antropológica, arqueológica e histórica. Su objetivo es proporcionar desarrollo, defensa, preservación, conservación y difusión del patrimonio cultural del país.

La inversión total de la Comuna 10 de La Candelaria para el periodo 2012-2015 fue de 557.768 mil millones de pesos, fondos provenientes de Recursos Ordinarios, Presupuesto Participativo, Jornada de Vida y Equidad, y el Fondo Medellín Ciudad para la Vida (Figura 9).

Parte de estos 557,768 mil millones de pesos, asignados a la Comuna 10, representados en proyectos, también fueron articulados y complementados con inversión privada. Así, se conformó lo que se ha denominado la Intervención Integral del Centro, donde la administración municipal ejecutó proyectos por 1,650 billones de pesos, mientras quedan aún en etapa de estudio y diseño 419,275 mil millones de pesos.

\section{Discusión}

La noción de centro histórico utilizada para esta gestión ha sido integral. En ese sentido, entenderlo como ciudad ha sido de vital importancia. Otro factor muy relevante fue la falta de delimitación del centro, pieza clave en esta intervención, ya que el centro histórico es considerado el centro de la ciudad viva y no un lugar intangible.

Las políticas de ordenamiento territorial aplicadas en Colombia aparecieron en un momento importante, esto es, en medio de la crisis que sufrieron los centros históricos de dicho país en los años 90. Durante esta década, tuvo lugar una destrucción masiva entre los centros históricos a causa de intereses personales. Los planes de ordenamiento, en principio, comenzaron estando ligados al desarrollo económico. Esta política malinterpretada fue una gran destructora de ciudades. Por ejemplo, si bien el uso de la tierra también es de carácter social, no fue sino hasta mediados de los años 90 que se creó una política orientada a cuidar el patrimonio, aunque ya se habían destruido algunos bienes de valor patrimonial. Nadie niega que Medellín ha sido transformada a lo largo de muchos años, pero varios aspectos habrían sido diferentes si, antes de que un plan de ordenamiento territorial estuviese ligado a lo económico, hubiese estado ligado principalmente a salvaguardar el patrimonio, es decir, la memoria histórica de la ciudad (Rodríguez, 2015).

Una fortaleza de Colombia es la fluida comunicación que permite la interacción entre sus gobiernos locales. Los municipios gozan de cierta autonomía, y es ahí de donde surgen los planes orientados a proteger sus bienes culturales. El gobierno respalda estas políticas y decisiones, permitiendo así que se realicen más planes de trabajo locales que, al mismo tiempo, estén dentro del Plan Nacional de Desarrollo. Esta descentralización del poder dinamiza el proceso de mejora de los centros históricos.

Es imposible imaginarse la gestión de Medellín sin el involucramiento de los POT, el patrimonio cultural y el urbanismo en Colombia. Como ya se especificó antes, el derecho urbanístico de los colombianos, que se consolidó dentro de los derechos colectivos al uso y goce del espacio público, gracias a la Ley de la Reforma Urbana, se convirtió en preocupación de orden constitucional, que ha recibido un amplio desarrollo jurisprudencial. De igual modo, la Constitución introdujo el derecho a una vivienda digna y el acceso a los servicios públicos, derecho que luego se vio plasmado en la gestión de Medellín, ciudad donde esta política ayudó a transformarla en un lugar donde se puede vivir con dignidad.

Cabe recalcar que, como ya se mencionó antes respecto del artículo de Diana Ramos, existen muchas veces problemáticas internas de colaboración mutua que los gobiernos locales tienen que afrontar. El POT y el PEMP, muchas veces, no consiguen llegar a 
buenos acuerdos: ¿cómo el POT incluye al PEMP si este solo se encarga del bien patrimonial?, y ¿cómo el PEMP se articula con el POT que no tiene lineamientos para cuidar el patrimonio edificado?

Sin embargo, al margen de todos estos desencuentros, las políticas culturales en Colombia aún siguen trabajando por el bien de sus ciudades históricas. A pesar de los problemas, es evidente la increíble transformación de esta ciudad, sin duda un ejemplo a seguir, por lo que resulta imprescindible aprender de sus éxitos y analizar sus fracasos.

En gran medida, el éxito de las gestiones realizadas en los centros históricos colombianos responde a la vinculación del ordenamiento territorial y el patrimonio cultural por encima de sus conflictos internos; en otras palabras, gran parte del éxito de Medellín está en su planificación. Los enfoques políticos y patrimoniales, unidos a las estrategias asumidas por el Estado, fueron una sólida base para las políticas locales de Medellín.

\section{Conclusión}

Una de las principales y fundamentales fortalezas del Plan Cultural de Medellín es que tiene el foro democrático muy presente. La cultura es un elemento que logra transversalizar las relaciones sociales, y relocalizarlas con sentidos originales, amplios y novedosos. Uno de los puntos clave en la intervención ha sido el trabajo integral en la recuperación del centro de Medellín, que le ha permitido convertirse en un centro sostenible. Para esto, fue fundamental la comprensión del centro histórico como ciudad, antes que solo como patrimonio. Todo eso, aliado a un plan de ordenamiento territorial y ligado a sus planes nacionales, ha terminado en éxito.

El proyecto de rehabilitación del centro histórico ha estado basado en la mejora del centro de la ciudad. Esto permitió que se trabaje en la mejora del espacio público, en un nuevo tipo de movilidad, en generar presencia institucional y en revitalizar el patrimonio. Para ello se tomó como instrumento normativo al PEMP. Las leyes sobre patrimonio han sido muy favorables para que los centros históricos sean protegidos, preservados y divulgados de manera exitosa. Sin embargo, muchas veces, las políticas han tenido desencuentros, como es el caso del POT y los PEMP, como se explicó anteriormente. A pesar de ello, se han realizado las actividades correspondientes y estas han terminado en un buen resultado.

El planeamiento de Medellín ha sido progresivo. Ha abarcado tres grandes periodos: 1990-2003, 2004-2011 y 2011-2015. En cada uno de estos se abordaron los problemas puntuales de cada época. El cuidado del centro y de la ciudad no son proyectos aislados, sino que es un tema latente en las agendas para los próximos años. Así lo demuestra el plan para Medellín en vigencia (2011-2021).

\section{Referencias}

Biblioteca Pública Piloto, (22 de abril del 2019). [Fotografía] Recuperado de: http://www.scielo.org. co/scielo.php?script=sci_nlinks\&ref=000122\&pid=S2145-132X201500020000800008\& $\mathrm{ln}$ $\mathrm{g}=\mathrm{es}$.

CEl. (2007). Informe nacional del sistema de cultura. Recuperado de http://www.oei.es/historico/cultura2/colombia/07.htm

Expansión. (2016). [Fotografía] Recuperado el noviembre de 2017 de Datosmacros.com: https:// www.datosmacro.com/demografia/poblacion/colombia

Colombia.com (17 de setiembre 2017). [Fotografía] Recuperado de: Recuperado de https:// www.colombia.com/colombia-info/informacion-general/geografia/

Colciencias (15 de noviembre 2018). Biodiversidad colombiana. Recuperado de https://www. colciencias.gov.co/portafolio/colombia-bio/quienes-somos 
devenir Vol. 6, N¹1, ENERO - JUNIO 2019, PP. 69-86 - ESTUDIOS I ISSN 2312-7562 I E-ISSN 2616-4949

UNIVERSIDAD NACIONAL DE INGENIERÍA, LIMA

doi: https://doi.org/10.21754/devenir.v6i11.634

Departamento Administrativo Nacional de Estadística (DANE), (2005). La visibilización estadística de los grupos étnicos colombianos. Recuperado de http://www.dane.gov.co/index.php/ terminos-de-uso/138-espanol/937-grupos-etnicos-prueba.

El Museo (2013). Foro para reflexionar el centro de Medellín. Recuperado de https://www.museodeantioquia.co/noticia/jornadas-para-reflexionar-el-centro-de-medellin/.Colombia.

Garavito, L. (2006). El origen del patrimonio como política en Colombia, y su relevancia para la interpretación de los vínculos entre cultura y naturaleza. OPERA, 6(6), 169-187. Recuperado de https://revistas.uexternado.edu.co/index.php/opera/article/view/1437

Humanaun Colombia (10 de febrero del 2019). [Fotografía]. Recuperado de: http://www.humanumcolombia.org

ICCROM (2012). Introduciendo a los jóvenes en la protección del patrimonio cultural y los centros históricos, una guía práctica para maestros en Colombia. Bogotá.

Melo, J(Ed.) (1996). Historia de Medellín (1 ed.). Ed. Bogotá: Compañía suramericana.

Ministerio de cultura. (2013). Política para la protección del patrimonio mueble. Recuperado de http://www.mincultura.gov.co/areas/patrimonio/publicaciones/Documents/Politica\%20 PCMU_Colombia.pdf.

Minvivienda (2014). Gobierno de Colombia, site oficial del Ministerio de Vivienda. Recuperado de http://www.minvivienda.gov.co/viceministerios/viceministerio-de-vivienda/espacio-urbano-y-territorial/renovaci\%C3\%B3n-urbana

Mincultura. (2016). Lista de bienes declarados bien de interés cultural del ámbito nacional. Recuperado de http://www.mincultura.gov.co/prensa/noticias/Documents/Patrimonio/listado\%20bienes\%20de\%20inter\%C3\%A9s\%20cultural\%20del\%20ambito\%20nacional.\%20 abril\%202016.pdf

PGIIC. (2015). Plan de gestión integral de la intervención del centro, Centro Metropolitano de Medellín, una construcción desde lo territorial, la planeación y el imaginario. Colombia, Gobierno regional de Antioquia, ISBN: en trámite.

Pinilla, Juan F. (2003). Evolución del sistema urbanístico colombiano. Reflexión pendiente desde el Derecho, Colombia. Recuperado de http://www.institutodeestudiosurbanos.info/dmdocuments/cendocieu/Especializacion_Mercados/Documentos_Cursos/Evolucion_Sistema_ Urbanistico-Pinilla_Juan-2003.PDF

Ramos, Diana C. (2016). El plan de ordenamiento territorial y el plan de manejo y protección del patrimonio en los centros históricos de Colombia: El caso Popayán. Devenir , ed.6, p.63-78.

Rodríguez, G. M. M. (2015). Estrategias de ordenamiento territorial en los centros históricos colombianos. Territorios. Recuperado de dx.doi.org/10.12804/territ32.2015.04

Tindo, (22 de abril del 2019). [Fotografía]. Recuperado de : https://sp.depositphotos. com/213393702/stock-photo-colombia-map-south-america-soft.html.

V Informe Nacional (2014). Biodiversidad de Colombia ante el convenio de diversidad biológica. Recuperado de https://issuu.com/pnudcol/docs/v_informe_nacional_de_biodiversidad.

WSJ (2013). Wall street magazine. Recuperado de https://www.semana.com/nacion/articulo/ medellin-ciudad-mas-innovadora-del-mundo/334982-3 